\title{
GÉIS POLIMÉRICOS CONTENDO AZUL DE METILENO COMO NOVAS FORMULAÇÕES PARA TERAPIA FOTODINÂMICA
}

\author{
L. A. G. da $\operatorname{COSTA}^{1}$, H. K. M. PEREIRA ${ }^{1}$, A. FONTES ${ }^{2}$, J. S. A. FALCÃO ${ }^{3}$, B. S. \\ SANTOS $^{1}$
}

${ }^{1}$ Departamento de Ciências Farmacêuticas, Universidade Federal de Pernambuco,

2 Departamento de Biofísica e Radiobiologia, Universidade Federal de Pernambuco

${ }^{3}$ Unidade Acadêmica de Saúde, Universidade Federal de Campina Grande,

E-mail para contato: lucasamadeusc2@hotmail.com

RESUMO - O azul de metileno (AM) em solução é utilizado na clínica como um eficiente fotossensibilizador para terapia fotodinâmica, ainda não existindo uma formulação tópica padronizada. O objetivo principal do trabalho é o desenvolvimento de um gel contendo AM para aplicação em terapia fotodinâmica. Neste estudo foram empregados géis a base de carbopol 940 (CBP) ou hidroxietilcelulose (HEC), adicionados de metilparabeno 0,15\%, trietanolamina q.s. pH 7, água destilada ou solução hidroalcoólica 10\%, ou solução de ureia 10\% q.s.p. e azul de metileno (1\%). Foram realizados testes de espalhabilidade das formulações, bem como sua caracterização óptica e a estimativa de eficiência de produção de espécies reativas de oxigênio (EROs). As formulações apresentaram coloração azul, $\mathrm{pH} \sim 7$ e espalhabilidade máxima $\sim 5000 \mathrm{~mm}^{2}$ para $C B P$ e $\sim 6500 \mathrm{~mm}^{2}$ para HEC. As melhores formulações no que se refere à geração de EROs foram CBP $940^{\circledR}$ com etanol $10 \%$ (61\% de geração) e HEC com água (60\% de geração). Estes valores correspondem a um aumento em torno de $23 \%$ com relação ao valor obtido na solução de AM (48\% de geração de EROs), sugerindo que a formulação propicia um ambiente físico-químico propício para a amplificação do efeito fotodinâmico.

Palavras-chave: fotossensibilizador; azul de metileno; formulação tópica; géis.

ABSTRACT - Methylene blue (MB) in aqueous solution used as a photosensitizer in clinical photodynamic therapy and there is not a standardized topical formulation yet. The main objective of this study is to develop a gel containing $M B$ for topic photodynamic therapy. We prepared carbomer 940 (CBP) or hydroxyethylcellulose (HEC) based gels containing also $0.15 \%$ methylparaben, triethanolamine q.s. $\mathrm{pH} \sim 7$, distilled water or $10 \%$ hydroalcoholic solution or $10 \%$ urea solution s.q.t. and methylene blue (1\%). Scatterability test was carried out for the formulations, as well as their optical characterization and the determination of reactive oxygen species (ROS). The formulations presented a blue color, $\mathrm{pH} 7$ and maximum spreadability close to $5000 \mathrm{~mm}^{2}$ for CBP and $6500 \mathrm{~mm}^{2}$ for HEC in water. The best formulations, in terms of generation of ROS 
were $\mathrm{CBP}$ with ethanol $10 \%(61 \% \mathrm{ROS})$ and of $\mathrm{HEC}$ with $10 \% \mathrm{H}_{2} \mathrm{O}(60 \% \mathrm{ROS})$, presenting a 23\% relative to the solution of $M B$ (48\% ROS production), suggesting that the formulation provides a favorable chemical physical environment for the amplification of the photodynamic effect.

Keywords: photosensitizer; methylene blue; topic formulation; gels.

\section{INTRODUÇÃ̃O}

Terapia fotodinâmica é definida como uma modalidade terapêutica que utiliza reações fotoquímicas para destruição seletiva de células e/ou tecido. Os fotossensibilizadores (FS), administrados de forma exógena ou presentes de forma endógena, são ativados pela luz e transferem sua energia para o oxigênio molecular presente no meio, gerando espécies reativas de oxigênio (EROs) que induzem morte celular (ISSA, MANELA-AZULAY, 2010). O azul de metileno (AM) é um fotossensibilizador que vem sendo empregado no tratamento de infecções bucais, periodontite e descontaminação endodôntica, sendo também relatado o uso no tratamento de alguns tipos de cânceres e micoses ungueais (TARDIVO et al., 2005). O AM possui ainda, boa eficácia de geração de ${ }^{1} \mathrm{O}_{2} \mathrm{e}^{3} \mathrm{O}_{2}$ (em torno de $50 \%$ ) segundo Nunez et al. (2013). Uma das preparações mais comuns de uso em clínica é solução de azul de metileno com concentrações entre 1 e $2 \%(\mathrm{~m} / \mathrm{v})$. Neste sentido, este trabalho visa o desenvolvimento de uma formulação simples na forma gel para aumento do tempo de contato do FS com o ponto de aplicação e, portanto, aumento da eficácia da terapia fotodinâmica tópica. Sendo assim, os objetivos do trabalho são: (i) preparar formulações semissólidas a base de gel para uso tópico contendo azul de metileno com concentração terapêutica; (ii) determinar $\mathrm{pH}$ e a espalhabilidade das formulações; (iii) caracterizar os sistemas obtidos através de espectroscopia de absorção, emissão e excitação e (iv) comparar o rendimento de geração de EROs do AM em solução e após sua incorporação em formulação gel.

\section{MATERIAIS E MÉTODOS}

Resumidamente, na preparação do gel carbopol (CBP) com $\mathrm{pH} \sim 7,0$ utilizou-se carbopol $940^{\circledR}$ a $1 \%$, metilparabeno a $0,15 \%$, trietanolamina q.s. $\mathrm{pH} 7$ e água destilada q.s.p., obtendose um sistema homogêneo a partir de mistura manual vigorosa. Para o gel de hidroxietilcelulose (HEC ou natrosol) foi empregada concentração de $2 \%$ no lugar do carbopol $940^{\circledR}$, mantendo-se os demais componentes. O azul de metileno foi veiculado nos géis para uma concentração final de $50 \times 10^{-6}$ mol.L ${ }^{-1}$ (para ensaios de geração de EROs) ou 1\% (para ensaios de propriedades físico-químicas). Todos os valores em $\%$ representam $\% \mathrm{~m} / \mathrm{v}$ no que se refere às proporções de componentes. Ensaios foram aplicados alterando-se fatores experimentais, dentre eles a concentração do AM na formulação e adição de adjuvantes farmacotécnicos, como ureia a 10\% e etanol (EtOH) também a 10\% com o propósito de verificar interações de possíveis agentes de permeação. As formulações foram armazenadas a $\mathrm{T}=4$ a $10{ }^{\circ} \mathrm{C}$ e protegidas da luz. Os testes de detecção de EROs foram realizados tanto na solução quanto nas formulações utilizando-se a metodologia descrita por Krajlic \& Mohsni (1978), em que as EROs geradas diminuem indiretamente a absorbância da N,N-dimetil-paranitrosoanilina (RNO), sendo sua absorbância avaliada no comprimento de onda em $\lambda=440$ $\mathrm{nm}$. A degradação foi feita utilizando-se laser MMOptics de fluência $33 \mathrm{~J} / \mathrm{cm}^{2}$ para $10 \mathrm{~s}$ de irradiação, comprimento de onda em $660 \mathrm{~nm}$ e com frequência de irradiação em intervalos de $20 \mathrm{~s}$ ou $1 \mathrm{~min}$ entre cada leitura até totalizar $5 \mathrm{~min}$. A leitura dos espectros de absorção e 
emissão/excitação eletrônica foi feita através do espectrofotômetro Lambda 650 UV/Vis e do fluorímetro modelo LS55, ambos da Perkin Elmer. O cálculo da porcentagem de geração de EROs equivale a $100-\%$ RNO consumido (denominado RNOc) o qual é estimado experimentalmente pelo cálculo: (absorbância final do FS x 100)/ (absorbância inicial do FS). A determinação do $\mathrm{pH}$ foi realizada diretamente na preparação através da utilização de fitas de $\mathrm{pH}$ e a espalhabilidade foi realizada de acordo com metodologia descrita por Zanin et al. (2001), em que uma massa da formulação foi submetida ao peso de placas de vidro sucessivamente e a área ocupada, calculada.

\section{RESULTADOS E DISCUSSÃO}

Todas as formulações apresentaram-se azuis, típicas do AM e os valores de $\mathrm{pH}$ apresentaram-se em torno de 7 para todas as formulações, inclusive as contendo ureia ou etanol a $10 \%$. No que se refere à espalhabilidade, não houveram alterações significativas comparadas com a do placebo, apresentando-se em torno de $5000 \pm 231 \mathrm{~mm}^{2}$ para formulação de CBP e em torno de $6500 \pm 40 \mathrm{~mm}^{2}$ para formulação de HEC. Foram observados deslocamentos (ca. $6 \mathrm{~nm}$ ) no máximo de emissão do $\mathrm{AM}\left(\lambda_{\text {máx }}(\mathrm{AM})=700 \mathrm{~nm}\right)$ após sua incorporação nas formulações, sugerindo interação entre os géis e o ativo (SANTOS, 2015), porém sem correlação direta com a capacidade de geração de EROs. Todas as seis formulações (e suas reproduções) apresentaram-se estáveis com a presença do ativo. Observou-se que nos sistemas $\mathrm{CBP} / \mathrm{EtOH}$ e $\mathrm{HEC} / \mathrm{H}_{2} \mathrm{O}$ (Tabela 1) foram obtidos os melhores resultados na produção de EROs. Sugere-se que este aumento decorra de uma distribuição efetiva do ativo na malha do gel, minimizando sua dimerização (NUÑEZ et al, 2015), citado como um dos processos responsáveis por reduzir a eficiência do AM. A redução da eficiência de produção de EROs nas formulações ocorre mais extensivamente nas amostras incorporadas de ureia, o que sugere alguma interferência desta espécie no mecanismo de formação das espécies reativas.

Tabela 1 - Comparação entre a capacidade de produção de EROs das diferentes formulações.

\begin{tabular}{|c|c|c|c|}
\hline Sistema/Aditivo & \multicolumn{3}{c|}{ Produção de EROs (\%) } \\
\hline $\boldsymbol{A M}(\mathbf{1 \% )} \boldsymbol{e m}$ & $\mathrm{H}_{2} \mathrm{O}$ & EtOH & Ureia \\
\hline Solução & $48,0 \pm 4,1$ & $64,0 \pm 0,5$ & $56,0 \pm 3,8$ \\
\hline Gel CBP 1,0\% & $37,0 \pm 0,9$ & $61,0 \pm 4,4$ & $40,0 \pm 2,5$ \\
\hline Gel HEC 2,0\% & $60,0 \pm 1,7$ & $56,0 \pm 0,9$ & $39,0 \pm 1,6$ \\
\hline
\end{tabular}

\section{CONCLUSÃO}

Os resultados apontam que em todas as formulações preparadas no presente estudo, a incorporação de AM foi realizada com sucesso, mas que em duas (CBP com EtOH 10\% e HEC $10 \%$ em $\mathrm{H}_{2} \mathrm{O}$ ) houve um aumento 23\% com relação ao valor obtido na solução de AM (48\% de geração de EROs), sugerindo que a formulação propicia um ambiente físico-químico adequado para a amplificação do efeito fotodinâmico. Os resultados sugerem certo grau de interação entre o AM e a malha polimérica e que o aumento da 
produção de EROs pode decorrer da reduzida mobilidade das moléculas tornando o processo de dimerização do AM ineficiente. Estudos estão sendo realizados para otimização das formulações com maior geração de EROs, levando em conta a padronização dos parâmetros físico-químicos, bem como sua aplicação em experimentos envolvendo inativação fotodinâmica.

\section{REFERÊNCIAS}

ISSA MCA, MANELA-AZULAY, M. 2010. Terapia fotodinâmica: revisão da literatura e documentação iconográfica. An Bras Dermatol. 85(4):501-11.

KRALJIC; MOHSNI, S. El. 1978. A new method for the detection of singlet oxygen in aqueous solution. Photochem. Photobiol. 28, 577-581.

NUÑEZ, S. C.; RIBEIRO, M. S.; GARCEZ, A. S. 2013. Princípios da terapia fotodinâmica. Terapia Fotodinâmica antimicrobiana na odontologia, p.312.

NUÑEZ, C.S., YOSHIMURA, T.M., RIBEIRO, M.S., JUNQUEIRA, H.C., MACIEL, C., COUTINHO-NETO, M.D., BAPTISTA, M.S. (2015) Urea enhances the photodynamic efficiency of methylene blue, J. Photochem. Photobiol. B

TARDIVO, J. P. et al. 2005. Methylene blue in photodynamic therapy: From basic mechanisms to clinical applications. Photodiag. Photodynamic Ther. 2(3), 175-191.

SANTOS, L. B. M. Incorporação de Zincoporfirina e Azul de Metileno em Gel e Avaliação para Uso em Terapia Fotodinâmica. 2016. 66 f. Dissertação (Mestrado) - UFPE, Recife, 2016.

ZANIN, S. M. W. et al. 2001. Parâmetros físicos no estudo da estabilidade das emulsões. Revista Visão Acadêmica, 2(2) 47-58.

\section{AGRADECIMENTOS}

Agradeço à minha mãe, sem a qual não seria quem eu sou. Depois à minha orientadora $\mathrm{e}$ a todos os demais profissionais que me auxiliaram nesse caminho que estou a percorrer. Agradeço à UFPE pela bolsa de estudos. Este trabalho faz parte de um projeto financiado pelo CNPq através do Instituto de Ciência e Tecnologia em Fotônica (INCT-INFo). 